\title{
Promotion and cyclic sieving on families of SSYT
}

\author{
Per Alexandersson, Ezgi Kantarci Oğuz and Svante Linusson
}

\begin{abstract}
We examine a few families of semistandard Young tableaux, for which we observe the cyclic sieving phenomenon under promotion.

The first family we consider consists of stretched hook shapes, where we use the cocharge generating polynomial as CSP-polynomial.

The second family contains skew shapes, consisting of disjoint rectangles. Again, the charge generating polynomial together with promotion exhibits the cyclic sieving phenomenon. This generalizes earlier results by B. Rhoades and later B. Fontaine and J. Kamnitzer.

Finally, we consider certain skew ribbons, where promotion behaves in a predictable manner. This result is stated in the form of a bicyclic sieving phenomenon.

One of the tools we use is a novel method for computing charge of skew semistandard tableaux, in the case when every number in the tableau occurs with the same frequency.
\end{abstract}

\section{Contents}

1 Introduction . . . . . . . . . . . . . . . . . . . . . 248

1.1 Cyclic sieving . . . . . . . . . . . . . . . 248

1.2 Main results . . . . . . . . . . . . . . . . . . . . . . 248

2 Preliminaries . . . . . . . . . . . . . . . . . 250

2.1 Semistandard tableaux and Jeu-de-taquin promotion . . . . . . . 250

2.2 Charge, cocharge and Kostka-Foulkes polynomials . . . . . . . . 252

3 Promotion on stretched hooks . . . . . . . . . . . . . . . . . . . 254

3.1 Plane partitions, Gelfand-Tsetlin polytopes, and cyclic sieving . . . 257

3.2 Discussion and background . . . . . . . . . . . . 260

4 Promotion and cyclic sieving on skew shapes . . . . . . . . . . . . 261

4.1 Disjoint union of rectangles . . . . . . . . . . . . . . 263

5 Bi-cyclic sieving on ribbon $\mathrm{SYT} \ldots \ldots \ldots \ldots \ldots$

6 Discussion . . . . . . . . . . . . . . . . . . . . . 271

References . . . . . . . . . . . . . . . . . . . . . . . . 272 


\section{Introduction}

The cyclic sieving phenomenon has been studied extensively since its introduction in 2004 [RSW04]. Briefly, this phenomenon relates a cyclic group action on a set of combinatorial objects with a $q$-analog of the cardinality of the set evaluated at roots of unity.

\subsection{Cyclic sieving}

The notion of cyclic sieving is defined as follows.

Definition 1. (Cyclic sieving, Reiner-Stanton-White [RSW04]) Let $X$ be a set of combinatorial objects and $C_{n}$ be a cyclic group of order $n$ acting on $X$. We say that the triple $\left(X, C_{n}, f(q)\right)$ exhibits the cyclic sieving phenomenon $(C S P)$, if for all $d \in \mathbb{Z}$,

$$
\left|\left\{x \in X: g^{d} \cdot x=x\right\}\right|=f\left(\xi^{d}\right)
$$

where $\xi$ is a primitive $n^{\text {th }}$ root of unity and $f(q) \in \mathbb{Z}[q]$.

Some relevant CSP-triples are listed in Table 1, together with the new results from this article.

One particular instance of CSP is given by the set of semistandard Young tableaux of a fixed rectangular shape under the promotion operation, where the corresponding polynomial is a $q$-analog of the $q$-hook-content formula (see B. Rhoades [Rho10a]). Rhoades' result was refined in [FK13] where cyclic sieving on rectangular SSYT with a fixed content vector was considered.

In a recent preprint, [APRU20], it is proved that for a fixed skew shape $\lambda / \mu$, there exists some cyclic group action $C_{n}$ of order $n$, such that

$$
\left(\operatorname{SYT}(n \lambda / n \mu), C_{n}, f^{n \lambda / n \mu}(q)\right)
$$

is a CSP-triple, where $f^{n \lambda / n \mu}(q)$ is a skew Kostka-Foulkes polynomial. Note that when $\mu=\varnothing, f^{n \lambda}(q)$ can be computed using the $q$-hook formula. The notion of multiplying every part in a partition with a factor is commonly referred to as stretching. It is therefore natural to explore situations similar to (2). Geometrically, stretching corresponds to dilation of the Gelfand-Tsetlin polytope associated with the skew shape $\lambda / \mu$.

\subsection{Main results}

There has been less research on non-rectangular shapes where promotion usually has a very high order, which makes it unlikely to find nice instances of CSP. 
One case in which promotion behaves nicely is the case of hook shapes. Hook semistandard tableaux have been considered in [BMS14], [OP19] and [PSV16]. In this paper, we consider certain semistandard Young tableaux where the shape is a stretched hook, i.e., of the form $\lambda=\left((a+1) n, n^{b}\right)$ for some positive integers $a$ and $b$.

Our first main theorem is as follows:

Theorem 2. (Corollary 26 below) Let $\lambda=\left((a+1) n, n^{b}\right)$, and let $\nu=n^{a+b+1}$. Then

$$
\left(\operatorname{SSYT}(\lambda, \nu),\langle\partial\rangle, q^{-n\left(\begin{array}{c}
b \\
2
\end{array}\right)} \widetilde{K}_{\lambda, \nu}(q)\right)
$$

exhibits the cyclic sieving phenomenon, where

$$
\widetilde{K}_{\lambda, \nu}(q)=\sum_{T \in \operatorname{SSYT}(\lambda, \nu)} q^{\operatorname{cc}(T)}=q^{n\left(\begin{array}{c}
b \\
2
\end{array}\right)} \prod_{i=1}^{a} \prod_{j=1}^{b} \frac{[i+j+n-1]_{q}}{[i+j-1]_{q}} .
$$

Here $\widetilde{K}_{\lambda, \nu}(q)$ is a modified Kostka-Foulkes polynomial, given as a cochargegenerating function (it is also possible to use the traditional Kostka-Foulkes polynomial accompanied by a different power of $q$, see Lemma 10).

We prove this result using an equivariant map from stretched hook semistandard Young tableaux to a set of rectangular plane partitions, where promotion corresponds to a product of certain toggle operations. We then use the fact that plane partitions together with such toggles exhibit cyclic sieving, see [SW20] (the case of order ideals of $2 \times[n]$ posets was considered earlier in [RS12]). We refer the reader to [AKLM05] and [Hop20a] for some related open problems on plane partitions and cyclic sieving.

We also show cyclic sieving on skew shapes consisting of a disjoint union of rows under the promotion operation. For $\nu \vdash m$ and $n \geq 1$, let $\operatorname{SM}(\nu, n)$ be the set of skew semistandard Young tableaux where the shape is a disjoint union of $\ell(\nu)$ rows $j$ with the length of row $j$ is equal to $n \nu_{j}$, and the content is given by $n^{m}$, that is, there are $n$ boxes with label $j$, for each $j=1, \ldots, m$. Note that in the case $n=1$ we simply get the standard tableaux of the shape described.

Theorem 3. (Theorem 31 below) The triple

$$
\left(\operatorname{SM}(\nu, n),\langle\partial\rangle, K_{\lambda / \mu, n^{m}}(q)\right)
$$

has the cyclic sieving property, where $K_{\lambda / \mu, n^{m}}(q)$ is a Kostka-Foulkes polynomial.

In [FK13], a cyclic sieving phenomenon involving $K_{a^{b}, \gamma}(q)$ is proved, where promotion acts on the set $\operatorname{SSYT}\left(a^{b}, \gamma\right)$. This result refines the earlier instance of CSP given in [Rho10a]. In Theorem 37, we generalize the result by FontaineKamnitzer to the case where promotion acts on skew shapes consisting of a disjoint 
Table 1 . The group action is given by $k$-promotion, $\partial$, or an appropriate power of it, depending on the rotational symmetry of the content composition. The action $\partial^{\dagger}$ is defined via so-called "toggles", but can be mapped in an equivariant manner to promotion on rectangular SSYT.

\begin{tabular}{|c|c|c|c|}
\hline Set & Group & Polynomial & Ref. \\
\hline Binary words, $\mathrm{BW}(2 n, n)$ & rot & {$\left[\begin{array}{c}2 n \\
n\end{array}\right]_{q}$} & {$[\mathrm{RSW04]}$} \\
\hline Words, $W\left(k n, n^{k}\right)$ & rot & {$\left[\begin{array}{c}k n \\
n, n, \ldots, n\end{array}\right]_{q}$} & [RSW04] \\
\hline Rectangular, $\operatorname{SSYT}\left(a^{b}\right)$ & $\partial$ & $q^{*} \mathrm{~s}_{a^{b}}\left(1, \ldots, q^{k-1}\right)$ & [Rho10a] \\
\hline Rectangular, $\operatorname{SSYT}\left(a^{b}, \gamma\right)$ & $\partial^{d}$ & $q^{*} K_{a^{b}, \gamma}(q)$ & [FK13] \\
\hline Hooks $\operatorname{SSYT}\left(\left(n-m, 1^{m}\right), \gamma\right)$ & $\partial^{d}$ & {$\left[\begin{array}{c}n z(\gamma)-1 \\
m\end{array}\right]_{q}$} & {$[\mathrm{BMS} 14]$} \\
\hline Plane partitions & $\partial^{\dagger}$ & $\prod_{\substack{1 \leq i \leq a \\
1 \leq j \leq b}} \frac{[i+j+n-1]_{q}}{[i+j-1]_{q}}$ & {$[\mathrm{SW} 20]$} \\
\hline Matrices $M\left(n \nu, n^{m}\right)$ & rot & $\sum_{\lambda \vdash m n} K_{\lambda, n} m(q) K_{\lambda, n \nu}(1)$ & [Rho10b] \\
\hline Stretched hooks & $\partial$ & $\prod_{\substack{1 \leq i \leq a \\
1 \leq j \leq b}} \frac{[i+j+n-1]_{q}}{[i+j-1]_{q}}$ & Corollary 26 \\
\hline Disjoint rows & $\partial$ & $K_{n \lambda / n \mu, n|\lambda / \mu|}(q)$ & Theorem 31 \\
\hline Disjoint rectangles & $\partial^{d}$ & $K_{\left(a_{1}^{b_{1}}\right) \oplus \ldots \oplus\left(a_{r}^{b_{r}}\right), \gamma}(q)$ & Theorem 37 \\
\hline Certain two-row ribbon & $\partial$ & {$\left[\begin{array}{c}n \\
b\end{array}\right]_{t}-[n]_{t}+[n-1]_{q}$} & Corollary 41 \\
\hline Certain three-row ribbon & $\partial$ & {$[n-2]_{t}+(n-3)[n-1]_{q}$} & Theorem 44 \\
\hline
\end{tabular}

union of rectangles. As it might be expected, the polynomial for the CSP is a Kostka-Foulkes polynomial indexed by a skew shape and a composition.

In Section 5, we study promotion on two families of ribbon shapes, where the order of promotion is nice. We prove two instances of bicyclic sieving.

At the end we provide a discussion on some open problems and give a few examples of ribbon shapes where the order of promotion is not so well-behaved.

Acknowledgement. The authors thank an anonymous referee for improving the exposition. The authors are grateful for the Institut Mittag-Leffler program on Algebraic and Enumerative combinatorics, spring 2020, funded by Swedish Research Council (Vetenskapsrådet), grant 2016-06596.

The first author has been funded by Knut and Alice Wallenberg Foundation (2013.03.07), and by the Swedish Research Council, grant 2015-05308. The second and third author have been funded by Swedish Research Council, grants 621-20144780 and 2018-05218.

\section{Preliminaries}

\subsection{Semistandard tableaux and Jeu-de-taquin promotion}

With every partition $\lambda=\left(\lambda_{1}, \lambda_{2}, \ldots, \lambda_{k}\right)$, we associate a Young diagram, an array with $\lambda_{i}$ boxes in row $i$. Given two partitions $\lambda$ and $\mu$ with $\mu \subset \lambda$, the skew-diagram 
$\lambda / \mu$ is given by the cells in $\lambda$ that are not in $\mu$. A semistandard Young tableau of shape $\lambda$ (or $\lambda / \mu$ ) is a filling of its boxes with positive integers such that each row is weakly increasing from left to right and each column is strictly increasing from top to bottom. We denote the set of semistandard tableaux of shape $\lambda$ by $\operatorname{SSYT}(\lambda)$. A semistandard tableau that contains each of the numbers from 1 to $n$ exactly once is called standard. The content of a tableau $T$ is the composition $\left(\nu_{1}, \nu_{2}, \ldots, \nu_{n}\right)$ where $\nu_{i}$ is given by the number of occurrences of $i$ in $T$. We denote the set of all semistandard Young tableaux of shape $\lambda$ and content $\nu$ by $\operatorname{SSYT}(\lambda, \nu)$.

With any tableau $T$, we associate a reading word denoted $\operatorname{rw}(T)$ : a listing of its entries row by row, left to right, bottom to top. Note that if $T$ is standard, its reading word is a permutation of $n$.

Example 4. A semistandard tableau of shape $(5,2,2)$ and its reading word.

$$
T=\begin{array}{|l|l|l|l|l|}
\hline 1 & 1 & 2 & 3 & 4 \\
\hline 2 & 3 & &
\end{array} \quad \quad \quad \quad r w(T)=342311234 .
$$

We act on semistandard Young tableaux by jeu-de-taquin promotion (also called $K$-promotion),introduced in [Sch63] and [Sch72]. We shall follow the definition given in [BMS14]. Our definition differs from theirs in the sense that we start with removing the smallest entries and decrement, whereas they start with removing the largest entries and increment. In practice, this corresponds to taking the inverse of the operation and does not make a difference in the context of cyclic sieving. Throughout this paper, we will refer to this operation simply as promotion, denoted by $\partial$.

Jeu-de-taquin promotion works as follows: First all entries on $T$ labeled 1 are replaced by dots. Then the dots are moved to the outside corners by repeatedly changing places with the smaller of the entries to the right or below (If both are equal, exchange is made with the entry below to maintain tableau rules). Once no more exchanges are possible, all numbers are decremented by one, and dots are replaced by $n$, as seen in the following example.

Example 5. Jeu-de-taquin promotion

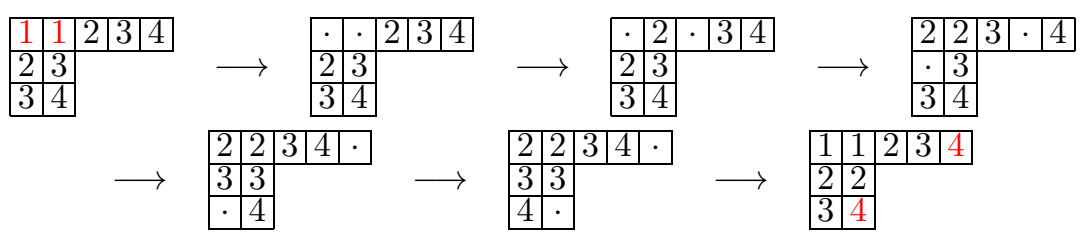




\subsection{Charge, cocharge and Kostka-Foulkes polynomials}

Definition 6. For a word $w$ we define the major index of $w$ as the sum $\operatorname{maj}(w):=\sum_{d \in \operatorname{Des}(w)} d$. If the word $w$ is also a permutation, we can look at its charge charge $(w):=\operatorname{maj}\left(\operatorname{rev}\left(\pi^{-1}\right)\right)$ and cocharge $\operatorname{cc}(w):=\left(\begin{array}{l}\ell \\ 2\end{array}\right)-\operatorname{charge}(w)$, where $\ell$ is the length of $w$.

Let $w$ be a word whose content is given by a partition $\left(v_{1}, \ldots, v_{n}\right)$. Then $w$ has $v_{1}$ standard subwords, defined as follows: To obtain the first standard subword, we start with rightmost 1 and proceed to the left to find a 2 then a 3 etc. all the way up to $\ell$, looping around when necessary. These letters, in their original order, make up the first standard subword $w_{1}$. Deleting the used letters gives us another word whose content is a partition, although the length may differ. We repeat the process to get the second standard subword, and then keep repeating till no letters are left and we got all $v_{1}$ standard subwords. Note that each subword is a permutation, giving us a way to extend the definition of charge and cocharge to the word $w$ by setting charge $(w):=\sum_{i} \operatorname{charge}\left(w_{i}\right)$ and $\operatorname{cc}(w):=\sum_{i} \operatorname{cc}\left(w_{i}\right)$.

In this work we focus on the case where $w$ is the reading word of a tableaux.

Example 7. The tableau $T \in \operatorname{SSYT}(9,3,3)$ below has the standard subwords $w_{1}=35214, w_{2}=42135, w_{3}=31245$ and charge $(\operatorname{rw}(T))=2+4+7=13, \operatorname{cc}(\operatorname{rw}(T))=8+$ $6+3=17$.

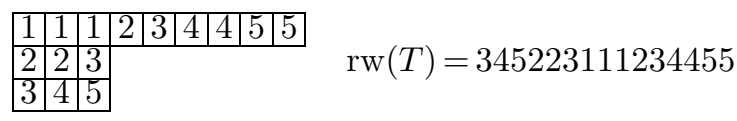

We shall also use the following alternative method of computing cocharge. Consider a permutation $\pi \in S_{\ell}$. For any $j \leq \ell$, define the cocharge value of $j$, denoted $c c(\pi, j)$ as follows:

$$
c c(\pi, j):= \begin{cases}0 & \text { if } j=1 \\ c c(\pi, j-1) & \text { if } j-1 \text { appears to the left of } j \text { in } \pi \\ c c(\pi, j-1)+1 & \text { otherwise. }\end{cases}
$$

The cocharge of $\pi$ is given by is the sum of these values: $\operatorname{cc}(\pi)=\sum_{j} c c(\pi, j)$.

Example 8. (Computing cocharge) Let $\pi=(4,8,6,9,7,2,3,1,5)$. The cocharge value $c c(\pi, j)$ is written as the subscript of $j$ :

$$
4_{2}, 8_{4}, 6_{3}, 9_{4}, 7_{3}, 2_{1}, 3_{1}, 1_{0}, 5_{2} \text {. }
$$

The cocharge of $\pi$ is 20 , the sum of the subscripts. 
Lemma 9. Let $\pi \in S_{\ell}$, and let rot act by cyclic shift to the right. Then

$$
\operatorname{cc}(\pi)= \begin{cases}\operatorname{cc}(\operatorname{rot}(\pi))-1 & \text { if } \pi(\ell) \neq 1 \\ \operatorname{cc}(\operatorname{rot}(\pi))+\ell-1 & \text { if } \pi(\ell)=1\end{cases}
$$

In particular $\operatorname{cc}(\pi) \equiv \operatorname{cc}(\operatorname{rot}(\pi))-1 \bmod \ell$.

Proof. This follows immediately from the recursion in (4).

The charge and cocharge statistics on tableaux can be used to calculate a special pair of families of polynomials called the Kostka-Foulkes polynomials and the modified Kostka-Foulkes polynomials.

$$
\begin{aligned}
K_{\lambda / \mu, \nu}(q) & :=\sum_{T \in \operatorname{SSYT}(\lambda / \mu, \nu)} q^{\operatorname{charge}(\operatorname{rw}(T))} \\
\widetilde{K}_{\lambda / \mu, \nu}(q) & :=\sum_{T \in \operatorname{SSYT}(\lambda / \mu, \nu)} q^{\operatorname{cc}(\operatorname{rw}(T))}
\end{aligned}
$$

The two families are related via the relation:

$$
\widetilde{K}_{\lambda / \mu, \nu}(q)=q^{\varkappa(\nu)} K_{\lambda / \mu, \nu}\left(q^{-1}\right),
$$

where

$$
\varkappa(\lambda):=\sum_{j}\left(\begin{array}{c}
\lambda_{j}^{\prime} \\
2
\end{array}\right),
$$

for the conjugate partition $\lambda^{\prime}$. Since $\lambda_{j}^{\prime}$ is the number of boxes in the $j^{\text {th }}$ column of $\lambda$, we get $\varkappa(n \lambda)=n \varkappa(\lambda)$. Moreover, for $\lambda=\left(a+1,1^{b}\right)$ we have $\varkappa(\lambda)=b(b+1) / 2$.

Note that permuting the entries of the content partition does not change the Kostka-Foulkes polynomial. That is,

$$
K_{\lambda / \mu, \sigma(\nu)}:=K_{\lambda / \mu, \nu}
$$

for any permutation $\sigma$. Warning! We may compute a Kostka-Foulkes polynomial via charge on SSYT only when the content is a partition.

Lemma 10. Let $\xi$ be an $n^{\text {th }}$ root of unity, such that $K_{\lambda / \mu, n \nu}(\xi)$ is a real number. Then $\widetilde{K}_{\lambda / \mu, n \nu}(\xi)=K_{\lambda / \mu, n \nu}(\xi)$.

Proof. By using (5), we have

$$
\widetilde{K}_{\lambda / \mu, n \nu}(\xi)=\xi^{n \cdot \varkappa(\nu)} K_{\lambda / \mu, n \nu}\left(\xi^{-1}\right)=K_{\lambda / \mu, n \nu}(\xi) .
$$

In the last equality, we use the fact that $\xi^{-1}=\bar{\xi}$, and that $K_{\lambda / \mu, n \nu}(\xi)$ is real. 


\section{Promotion on stretched hooks}

The main results of this section are a new way of computing the charge of a word with rectangular content and bijection between stretched hooks and plane partitions which allows to prove cyclic sieving for stretched hooks.

We define the Kostka coefficient as $K_{\lambda \mu}:=K_{\lambda \mu}(1)=|\operatorname{SSYT}(\lambda, \mu)|$.

Given $a, b \geq 0$ and $n \geq 1$, let $\operatorname{SHST}(a, b, n)$ (stretched hook semistandard tableaux) be the set

$$
\operatorname{SHST}(a, b, n):=\left\{T \in \operatorname{SSYT}(\lambda, \mu) \mid \lambda=\left(n a+n, n^{b}\right), \mu=\left(n^{a+b+1}\right)\right\} .
$$

We let $k$-promotion $\partial$ act on $\operatorname{SHST}(a, b, n)$.

Example 11. The six tableaux in $\operatorname{SHST}(1,2,2)$ form two 3-cycles under promotion.

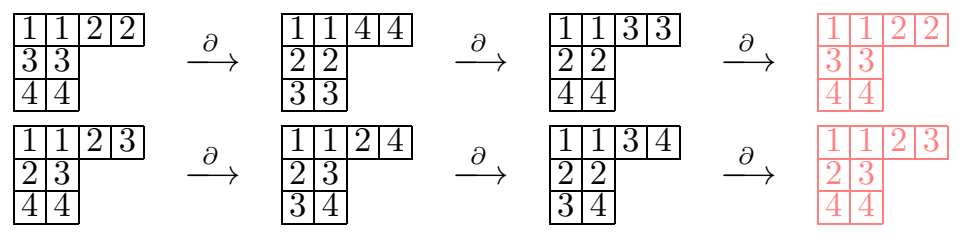

Note that in the example above, repeating promotion $a+b$ times lets us recover our original tableaux. Now we will show that this is always the case.

Proposition 12. (Order of promotion) For any $T \in \operatorname{SHST}(a, b, n)$, we have $\partial^{a+b}(T)=T$.

Proof. The first $n$ boxes in the first row of $T$ contain ones, which are replaced with twos after removal. Thus the promotion operation on stretched hook shapes is independent of the entries of the first row, as the total content is fixed. Promotion acts on the remaining $b \times n$ rectangle through promotion of entries from the alphabet $2,3, \ldots, a+b+1$. This is equivalent to doing promotion on rectangular semi standard Young tableaux filled with entries from the alphabet $[a+b]$, which has the property $\partial^{a+b}(T)=T$.

Deleting the first row and decrementing entries by one gives a bijection between $\operatorname{SHST}(a, b, n)$ and $\operatorname{SSYT}\left(n^{b}, a+b\right)$ that commutes with promotion, which we used in proving our proposition above. What makes the stretched hook shapes still interesting is the use of cocharge statistic and its relation with the promotion operation.

Let $|\operatorname{SHST}(a, b, n)|_{q}:=\sum_{T \in \operatorname{SHST}(a, b, n)} q^{\operatorname{cc}(T)}$. Note that this is a (modified) Kostka-Foulkes polynomial. 
Example 13. The six stretched hook semistandard tableaux given in Example 11 above have cocharges $6,10,8,7,8$, and 9 , respectively. The corresponding polynomial is

$$
|\operatorname{SHST}(1,2,2)|_{q}=q^{6}+q^{7}+2 q^{8}+q^{9}+q^{10}=q^{6}\left(1+q+q^{2}\right)\left(1+q^{2}\right) .
$$

Given a word $w$ on $[k]$ and an integer $j \geq 1$, consider the subword of $w$ consisting of entries in $\{j, j+1\}$. By repeatedly removing consecutive pairs $(j+1, j)$, we end up with a word of the form $j^{r}(j+1)^{s}$. We let $\delta_{j}(w)$ be the value of $s$, and set

$$
\delta(w):=\left(\delta_{1}(w), \delta_{2}(w), \ldots, \delta_{k-2}(w)\right)
$$

to be the depth sequence of $w$. The depth sequence gives a new way of computing the charge of any word with rectangular content.

Theorem 14. (Charge for rectangular content) Suppose $w$ is a word with content $n^{k}$. Then

$$
\operatorname{charge}(w)=\sum_{j<k} \delta_{j}(w)(k-j) .
$$

Proof. To calculate charge, we first divide $w$ into standard subwords, which in this case all have the same length $k$. We then proceed to calculate the charge of each subword. The contribution of a letter $j$ to charge just depends on whether it comes before or after $j+1$ in its subword. So, limiting our attention to $j$ s, we can see the subword selection process as an ordering on the $n$ entries labeled $j$, which then in order the first unclaimed $j+1$ to their left, looping around if necessary.

We shall now show that the total contribution of $j \mathrm{~d}$ to the charge is independent of the ordering. As the pairings with $j+1$ coming before $j$ do not contribute to the charge, we can use (8).

Consider an ordering and exchange the order of two consecutively ordered entries. There are two possiblities. They either pair up with their original $(j+1) \mathrm{s}$ in which case total charge does not change, or they swap. The latter can happen only if the relevant entries have one of the following four relative orderings:

$$
(j+1)(j+1) j j, \quad(j+1) j j(j+1), \quad j(j+1)(j+1) j, \quad j j(j+1)(j+1) .
$$

It is straightforward to verify that the change in order does not change the total contribution to charge.

Example 15. Note that the first row of the tableaux from Example 11 have depth sequences $(2,0,0),(0,0,2),(0,2,0)$ respectively. Their charges are 6,2 and 4 as expected. Note that promotion rotates the depth sequence in this example. We will next look at an easy way to calculate the depth sequence using only the first row, which will show that this is always the case. 
Theorem 16. Let $T \in \operatorname{SHST}(a, b, n)$. The depth sequence of the reading word of $T$ only depends on the first row of $T$. In particular, for each $j<a+b, \delta_{j}$ is equal to the number of $j+1 \mathrm{~s}$ on the first row.

Proof. Consider the entries $j$ and $j+1$ in the reading word. The $j+1$ s on the first row will not be matched to any $j$ s as they are at the end of the word, so $\delta_{j}$ is greater than or equal to the number of $j+1 \mathrm{~s}$ in the first row. We claim that any other $j+1$ will be paired up with $j$ under the algorithm, and therefore will not contribute to charge.

If we ignore the first row, we are left with $n$ columns of length $b$ each. For each column there are four possiblities: it can have a $j$, a $j+1$, both or neither. If it has a $j+1$ and a $j$, the $j$ will appear directly above the $j+1$, therefore it will be after $j+1$ in the reading word. Additionally, if we have $l$ columns with just $j+1$, we can have at most $n-l$ cells labeled $j$ in the first $n$ columns, so there are at least $l$ corresponding $j$ s on the first row, meaning all single $j+1$ s will be matched up as well.

Corollary 17. For $T \in \operatorname{SHST}(a, b, n)$ we have

$$
\begin{aligned}
\operatorname{charge}(T) & =n((a+b+2) a+1)-\Sigma(T) \quad \text { and } \\
\operatorname{cc}(T) & =n \frac{(b-a-1)(a+b+2)}{2}+\Sigma(T),
\end{aligned}
$$

where $\Sigma(T)$ is the sum of all entries of the first row.

Proof. Using Theorem 14 and 16 we get

$$
\begin{aligned}
\operatorname{charge}(T)+\Sigma(T) & =\sum_{j \geq 2} \delta_{j-1}(\operatorname{rw}(T))(a+b+1-(j-1))+n+\sum_{j \geq 2} \delta_{j-1}(\operatorname{rw}(T)) j \\
& =\sum_{j \geq 2} \delta_{j-1}(\operatorname{rw}(T))(a+b+2)+n=a n(a+b+2)+n,
\end{aligned}
$$

which gives the first equality. The reading word of $T$ has $n$ standard subwords of length $a+b+1$, which together with the definition of cocharge in Definition 6 gives

$$
\operatorname{cc}(T)=n\left(\begin{array}{c}
a+b+1 \\
2
\end{array}\right)-\operatorname{charge}(T) .
$$

The second equality follows.

The reader is now encouraged to compute the cocharge of the first tableau in Example 11 using both the definition and the formula above. The answer is 6 . 
Corollary 18. For $T \in \operatorname{SHST}(a, b, n)$ we have

$$
\delta \circ \partial(T)=\operatorname{rot}^{-1} \circ \delta(T) .
$$

That is, promotion rotates the depth sequence one unit to the left.

Proof. This follows as the action of promotion on the first row is just removing any 2 s, subtracting 1 from larger entries, and adding $a+b+1$ s to replace the $2 \mathrm{~s}$.

\subsection{Plane partitions, Gelfand-Tsetlin polytopes, and cyclic sieving}

A plane partition $\pi$ is a rectangular array with non-negative integer entries, such that rows are weakly increasing from left to right and columns are weakly decreasing from top to bottom.. Let $\mathcal{P} \mathcal{P}(a, b, n)$ denote the set of plane partitions within a $a \times b$-rectangle, with maximal entry at most $n$. A classical result due to MacMahon [Mac96, p. 659] states that the generating function for plane partitions is

$$
M_{q}(a, b, n):=\sum_{\pi \in \mathcal{P} \mathcal{P}(a, b, n)} q^{|\pi|}=\prod_{i=1}^{a} \prod_{j=1}^{b} \frac{[i+j+n-1]_{q}}{[i+j-1]_{q}} .
$$

Here $|\pi|$ denotes the sum of all entries in the plane partition.

A result due to R. Stanley [Sta01] implies that for $\lambda=b^{a}$, MacMahon's generating function is essentially a principal specialization of a rectangular Schur polynomial:

$$
M_{q}(a, b, n)=q^{-\varkappa(\lambda)} \mathrm{s}_{\lambda}\left(1, q, q^{2}, \ldots, q^{a+b-1}\right) .
$$

Definition 19. A Gelfand-Tsetlin pattern, or GT-pattern for short, is a triangular arrangement of non-negative integers with certain constraints. The triangular patterns are indexed as follows:

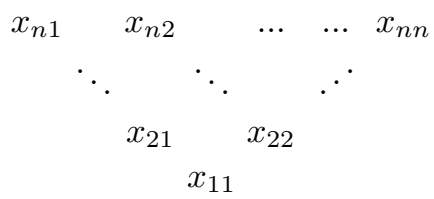

The entries must fulfill

$$
x_{i+1, j} \geq x_{i j} \quad \text { and } \quad x_{i j} \geq x_{i+1, j+1}
$$

for all values of $i, j$ where the indexing is defined.

There is a classical bijection between GT-pattern with top row equal to $\lambda_{1}, \ldots$, $\lambda_{\ell}$, and semistandard Young tableaux of shape $\lambda$ and entries $\leq \ell$. With this bijection, a tableau $T$ corresponds to a GT-pattern GT $(T)$, by letting the entries 
In general, the biggest charge in $\operatorname{SHST}(a, b, c)$ is obtained when the first row has content $n^{a+1}$ and is equal to $a(a+2 b+1) n / 2$.

\begin{tabular}{|l|l|l|l|l|l|l|l|l|l|l|l|}
\hline 1 & 1 & 1 & 1 & 5 & 5 & 5 & 5 & 6 & 6 & 6 & 6 \\
\hline 2 & 2 & 2 & 2 & & & & & & \\
\cline { 1 - 2 } 3 & 3 & 3 & 3 & & & & & & \\
\cline { 1 - 2 } 4 & 4 & 4 & 4 & & & & & & \\
\hline
\end{tabular}

$$
\begin{aligned}
& \begin{array}{llllll}
12 & 4 & 4 & 4 & 0 & 0
\end{array} \\
& \begin{array}{lllll}
8 & 4 & 4 & 4 & 0
\end{array} \\
& \begin{array}{llll}
4 & 4 & 4 & 4
\end{array} \\
& \begin{array}{lll}
4 & 4 & 4
\end{array} \\
& 44 \\
& 4
\end{aligned}
$$

Similarly, a maximal first row gives us the smallest possible charge, which is equal to 12 in the example above. The corresponding plane partition $\rho(T)$, is formed of all $4 \mathrm{~s}$, and has sum $|\rho(T)|=16$.

The maximum charge in $\operatorname{SHST}(a, b, c)$ is obtained when the first row has content $n^{a+1}$ and is equal to $a(a+2 b+1) n / 2$. In general, the smallest possible charge in $\operatorname{SHST}(a, b, c)$ is equal to $a(a+1) n / 2$, attained when the first row is filled with $n$ 1 followed by $n$ each of $b+2$ s to $b+a+1 \mathrm{~s}$. The corresponding plane partition has sum $|\rho(T)|=a b n$, giving us the total $a(a+2 b+1) n / 2$

Note that in both cases, the sum of the charge of the tableau and the entries on the corresponding plane partition is the same. Next, we will show that this is always the case.

Theorem 22. Let $T \in \operatorname{SHST}(a, b, n)$ and let $\rho(T)$ be its image under the bijection defined above. Then we have

$$
\begin{gathered}
\operatorname{charge}(T)+|\rho(T)|=\frac{n(a+2 b+1)(a)}{2} \\
\operatorname{cc}(T)-|\rho(T)|=\frac{n(b+1)(b)}{2}
\end{gathered}
$$

Proof. We will focus on the first identity, as the second follows directly from the first by plugging in charge $(T)+\operatorname{cc}(T)=\frac{n(a+b)(a+b+1)}{2}$.

We already showed that the identity holds if $\rho(T)$ is formed by $n$ s only. Assume (14) holds when the sum of the entries is larger than or equal to $M$. Consider a pattern with $|\rho(T)|=M-1$. As this is not the maximal case, there is an entry that we can increase by 1 without violating the rules. This operation corresponds to replacing the rightmost $k$ by $k+1$ in the top row and replacing a $k+1$ by $k$ in a row below, where the choice of $k$ and the place of the above entry depend on which coordinate we increase. This increases the sum of the first row by 1 , so by Corollary 17 the sum of charge $(T)$ and $|\rho(T)|$ stays the same.

An immediate result of this formula is the effect of exchanging $a$ and $b$ on the cocharge polynomial. If we flip the rectangle $\rho(T)$ around a horizontal line we 
obtain a rectangle still satisfying the inequalities inside a GT-pattern but with $a$ and $b$ interchanged.

Corollary 23. (Conjugation Symmetry) Flipping the rectangle $\rho(T)$ horizontally gives a bijection between $\operatorname{SHST}(a, b, n)$ and $\operatorname{SHST}(b, a, n)$, which preserves $|\rho(T)|$ and thus the cocharge. In particular, we have

$$
|\operatorname{SHST}(a, b, n)|_{q}=q^{\frac{n\left(a^{2}-a-b^{2}+b\right)}{2}}|\operatorname{SHST}(b, a, n)|_{q} .
$$

Also, combining the above result with Equation (9) gives us a way to calculate $|\operatorname{SHST}(a, b, n)|_{q}$ directly.

Corollary 24. (Kostka-Foulkes polynomials as plane partitions) We have the identity

$$
|\operatorname{SHST}(a, b, n)|_{q}=q^{n\left(\begin{array}{c}
b \\
2
\end{array}\right)} \prod_{i=1}^{a} \prod_{j=1}^{b} \frac{[i+j+n-1]_{q}}{[i+j-1]_{q}}=q^{n\left(\begin{array}{c}
b \\
2
\end{array}\right)} M_{q}(a, b, n) .
$$

The main result in [SW20] is the following instance of the cyclic sieving phenomenon on plane partitions, under an operation called toggles.

Theorem 25. (See $[\mathrm{SW} 20])$ Let $\tau$ act on $\mathcal{P} \mathcal{P}(a, b, n)$. Then

$$
\left(\mathcal{P P}(a, b, n),\langle\tau\rangle, M_{q}(a, b, n)\right)
$$

is a CSP-triple.

Combining this with Corollary 24 we get our first main result.

Corollary 26. (Cyclic sieving on stretched hook tableaux) Promotion acts on $\operatorname{SHST}(a, b, n)$ with order $a+b$. Furthermore, the triple

$$
\left(\operatorname{SHST}(a, b, n),\langle\partial\rangle, q^{-n\left(\begin{array}{c}
b \\
2
\end{array}\right)} \operatorname{SHST}_{q}(a, b, n)\right)
$$

is a CSP-triple.

Proof. Note that the action of promotion on any $T \in \operatorname{SHST}(a, b, n)$ is determined by its action on the filling of the rectangular shape obtained by deleting the first row of $T$. Our bijection with plane partitions matches the one described in [Hop20a, Appendix 1] where it is shown that the toggle operation is mapped to $\partial^{-1}$. The result follows as $q^{-n\left(\begin{array}{c}b \\ 2\end{array}\right)} \operatorname{SHST}_{q}(a, b, n)=M_{q}(a, b, n)$.

Note that the case where $n=1$ corresponds to a special case of [BMS14].

\subsection{Discussion and background}

The earliest reference connecting toggles and promotion is the article by A. Kirillov and A. Berenstein [KB96]. Later in [SW20] it is proved that that plane par- 
titions in an $a \times b$-box with max size $n$ exhibit CSP under piecewise linear toggles, without mentioning the connection with promotion. The connection between promotion, toggles and cyclic sieving is made explicit in S. Hopkins [Hop20a], where he studies plane partitions with additional symmetry (e.g., symmetric under transposition). He also discusses the connection with promotion and rowmotion on posets considered in [SW12].

Question 27. There is a promotion-type action on type $B$ minuscule poset ideals, see [Hop20b] and [RS12]. This is the same as a type of toggle on symmetric plane partitions, and there are $2^{n}$ such plane partitions. Can this action also be realized as an action on SSYT?

\section{Promotion and cyclic sieving on skew shapes}

For an integer partition $\nu \vdash m$, let $\operatorname{SM}(\nu, n)$ be the set of skew semistandard Young tableaux where the shape is a disjoint union of $\ell(\nu)$ rows with row $j$ of length $n \nu_{j}$, and the content is $n^{m}$. Given a tableau $T \in \operatorname{SM}(\nu, n)$, define an associated matrix $M(T)=\left[M_{i j}\right]_{i j}$ satisfying

$$
M_{i j}=\text { number of entries in row } i \text { of } T \text { with value } j .
$$

By construction we have

$$
\sum_{j \geq 1} M_{i j}=n \nu_{i} \quad \text { and } \quad \sum_{i \geq 1} M_{i j}=n
$$

Note that this a bijection between $\operatorname{SM}(\nu, n)$ and $\mathcal{M}\left(n \nu, n^{m}\right)$, the set of non-negative integer matrices with row sums given by $n \nu$ and column sums all equal to $n$. To a given matrix $M \in \mathcal{M}\left(n \nu, n^{m}\right)$, we associate a biword $W$ where each pair $\left(\begin{array}{l}i \\ j\end{array}\right)$ appears $M_{n+1-i, j}$ times and the entries are sorted lexicographically. This gives us a bijection between $\operatorname{SM}(\nu, n)$ and biwords of length $n|\nu|$ with the content of the top row equal to $\left(n \nu_{\ell}, \ldots, n \nu_{2}, n \nu_{1}\right)$ and the content of the bottom row equal to $(n, n, \ldots, n)$. Moreover, if $T$ is mapped to $W$ (via $M(T)$ ) then the reading word of $T$ is the bottom row of $W$.

Example 28. Below we give a $T \in \mathrm{SM}(211,3)$, the corresponding matrix $M(T)$, and the biword $W(T)$.

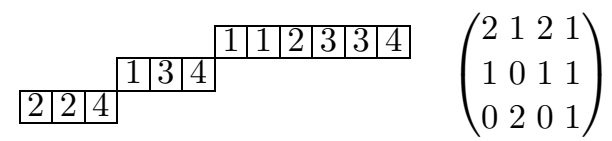

$\left(\begin{array}{llllllllllll}1 & 1 & 1 & 2 & 2 & 2 & 3 & 3 & 3 & 3 & 3 & 3 \\ 2 & 2 & 4 & 1 & 3 & 4 & 1 & 1 & 2 & 3 & 3 & 4\end{array}\right)$ 
Proposition 29. Let $\nu \vdash m$ and $n \geq 1$. The Robinson-Schensted-Knuth correspondence $(R S K)$ gives a bijection

$$
\begin{gathered}
\operatorname{SM}(\nu, n) \stackrel{\mathrm{RSK}}{\longrightarrow} \bigcup_{\lambda \vdash n m} \operatorname{SSYT}\left(\lambda, n^{m}\right) \times \operatorname{SSYT}(\lambda, n \bar{\nu}) \\
T \stackrel{\mathrm{RSK}}{\longrightarrow}(P, Q) .
\end{gathered}
$$

where the insertion algorithm is performed on the biword associated with $T$, and $\bar{\nu}$ denotes the reverse of $\nu$. Furthermore, charge $(T)=\operatorname{charge}(P)$.

Proof. Let $w$ be the bottom row of the biword associated with $T$, i.e., $w=$ $\mathrm{rw}(T)$. By definition, charge $(T)=\operatorname{charge}(w)$. Moreover, $w$ insert to the semistandard tableau $P$ under RSK (via the bumping procedure), so $w$ and $\operatorname{rw}(P)$ are Knuth-equivalent, so they have the same charge (see e.g. [But94]) implying charge $(T)=\operatorname{charge}(P)$.

We let the $q$-analogue of $|\operatorname{SM}(\nu, n)|$ be defined as

$$
|\operatorname{SM}(\nu, n)|_{q}:=\sum_{T \in \operatorname{SM}(\nu, n)} q^{\operatorname{charge}(T)} .
$$

By definition, $|\operatorname{SM}(\nu, n)|_{q}$ is the skew Kostka-Foulkes coefficient $K_{\lambda / \mu, n^{m}}(q)$, where $\lambda / \mu$ is the skew shape defined by the disjoint rows of lengths given by the parts of $n \nu$.

Corollary 30. Let $\nu \vdash m$ and $n \geq 1$. Then

$$
|\mathrm{SM}(\nu, n)|_{q}=\sum_{\lambda \vdash m n} K_{\lambda, n^{m}}(q) K_{\lambda, n \nu}(1)
$$

Proof. The identity follows from Proposition 29, and the fact that $K_{\lambda \nu}=$ $K_{\lambda \bar{\nu}}$.

Note that $K_{\lambda, \nu}(1)=K_{\lambda, \nu}$, the Kostka coefficient defined previously.

Theorem 31. (Cyclic sieving on stretched skew shapes with disjoint rows) For $\nu \vdash m$ and $n \geq 1$, let $\lambda / \mu$ to be the skew shape defined by the disjoint rows of lengths given by the parts of $n \nu$. Then the triple

$$
\left(\operatorname{SM}(\nu, n),\langle\partial\rangle, K_{\lambda / \mu, n^{m}}(q)\right)
$$

has the cyclic sieving property.

Proof. We first note that $\partial$ acting on $T \in \mathrm{SM}(\nu, n)$ simply corresponds to cyclic rotation of the columns one step left in $M(T)$. Hence, elements in $\operatorname{SM}(\nu, n)$ fixed under $\partial^{d}$ are in bijection with the matrices in $\mathcal{M}\left(n \nu, n^{m}\right)$ which are fixed under 
cyclic $j$ step rotation of their columns. A result by B. Rhoades [Rho10b, Thm. 1.3], with a suitable specialization, implies that

$$
\left(M\left(n \nu, n^{m}\right),\langle\text { cyclic rotation of columns }\rangle, \sum_{\lambda \vdash m n} K_{\lambda, n^{m}}(q) K_{\lambda, n \nu}(1)\right)
$$

is a triple which exhibits the cyclic sieving phenomenon. Together with Corollary 30, the theorem follows.

\subsection{Disjoint union of rectangles}

We shall now extend Theorem 31 to a larger class of skew tableaux formed by disjoint rectangles with arbitrary content.

In [Rho10a], B. Rhoades proved that promotion acting on rectangular semistandard Young tableaux of shape $a^{b}$ with entries bounded by $m$, gives an instance of cyclic sieving together with the polynomial $q^{*} \mathrm{~s}_{a^{b}}\left(1, q, \ldots, q^{m-1}\right)$. This was later refined in [FK13], where the content of the semistandard tableau was held fixed, and the CSP-polynomial is given by a Kostka-Foulkes polynomial multiplied by a power of $q$. In this subsection, we extend their result to skew shapes consisting of disjoint unions of rectangles. First, some additional notation. Let $\mu^{k}$ denote the partition obtained from $\mu$ by repeating each part $k$ times, so that if $\mu=1^{a_{1}} 2^{a_{2}} \ldots \ell^{a_{\ell}}$ then $\mu^{k}:=1^{k \cdot a_{1}} 2^{k \cdot a_{2}} \ldots \ell^{k \cdot a_{\ell}}$. We will use a similar notation for compositions where we simply concatenate the parts, e.g. $125^{3}=125125125$.

A skew shape with $k$ boxes is a $k$-ribbon if it is connected and does not contain a $2 \times 2$-arrangement of boxes. The head of a ribbon is the upper right-most box. A collection of $k$-ribbons form a horizontal strip if their union is a skew shape and their heads lie in different columns. A semistandard $k$-ribbon tableau of shape $\lambda / \mu$ and content $\nu$ is a sequence of skew shifted diagrams $\lambda_{1} / \mu \subset \lambda_{2} / \mu \subset \ldots \subset \lambda_{n} / \mu=\lambda / \mu$ where $\lambda_{1} / \mu$ is a horizontal ribbon strip containing $\nu_{1} k$-ribbons and each $\lambda_{i+1} / \lambda_{i+1}$ is a horizontal $k$-ribbon strip containing $\nu_{i} k$-ribbons.

We let $K_{\lambda / \mu, \nu}^{(k)}$ be the number of semistandard $k$-ribbon tableaux of shape $\lambda / \mu$ and content $\nu$. Note that when $k=1$, we recover the usual Kostka coefficient $K_{\lambda / \mu, \nu}$. Finally, if $\lambda / \mu$ is a ribbon with $k$ boxes, we let $\varepsilon_{k}(\lambda / \mu):=(-1)^{h-1}$ where $h$ is the number of rows of $\lambda / \mu$. For arbitrary skew shapes $\lambda / \mu$, we let

$$
\varepsilon_{k}(\lambda / \mu):=\prod_{j} \varepsilon_{k}\left(\lambda^{(j)} / \mu^{(j)}\right)
$$

where $\lambda^{(1)} / \mu^{(1)}, \lambda^{(2)} / \mu^{(2)}, \ldots$ is any partitioning of $\lambda / \mu$ with $k$-ribbons (the sign can be shown to be independent of the particular choice of partitioning) with the convention that $\varepsilon_{k}(\lambda / \mu)=0$ if no such partitioning exists. 
Example 32. The 3-ribbon tableaux counted by $K_{4422,1111}^{(3)}$ are the following six fillings,

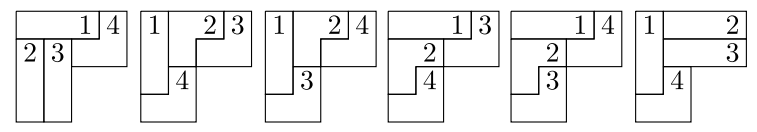

and the 3 -ribbon tableaux counted by $K_{4422,211}^{(3)}$ are given by

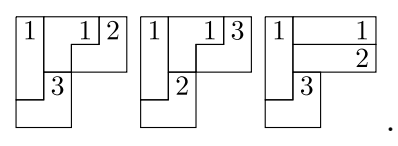

It is straightforward to verify that $\varepsilon_{3}(4422)=-1$, for example, as the last semistandard 3 -ribbon tableau gives a partitioning with four 3 -ribbons, with signs $(-1)^{3-1}$, $(-1)^{1-1},(-1)^{1-1}$, and $(-1)^{2-1}$, respectively.

It can be shown (see e.g., [APRU20]) that $\varepsilon_{k}(\lambda / \mu)$ can be given in terms of a skew character, but we do not need that here.

Theorem 33. (See [DLT94, p. 29]) Consider a skew shape $\lambda / \mu$ and a weak composition $\nu$. Let $\xi$ be a primitive $j^{\text {th }}$ root of unity. Then

$$
K_{\lambda / \mu, \nu}^{(j)}=(-1)^{|\nu|(j-1)} \varepsilon_{j}(\lambda / \mu) K_{\lambda / \mu, \nu^{j}}(\xi) .
$$

For example, $K_{4422,2^{3} 1^{3} 1^{3}}(q)$ is given by

$$
\begin{aligned}
& q^{25}+q^{24}+4 q^{23}+5 q^{22}+10 q^{21}+13 q^{20}+21 q^{19}+ \\
& 24 q^{18}+33 q^{17}+34 q^{16}+39 q^{15}+36 q^{14}+36 q^{13}+ \\
& 27 q^{12}+23 q^{11}+14 q^{10}+9 q^{9}+4 q^{8}+2 q^{7}
\end{aligned}
$$

and it evaluates to -3 at $q=e^{2 \pi i / 3}$. This is in agreement with the fact that $K_{4422,211}^{(3)}=3$, as we saw in Example 32. Note that basic properties of KostkaFoulkes polynomials allows us to reorder the content composition. For the example we have $K_{4422,2^{3} 1^{3} 1^{3}}(q)=K_{4422,211211211}(q)$.

We now recall the main result in [FK13].

Theorem 34. (Cyclic sieving on rectangular SSYT, fixed content) Let $\left(\gamma_{1}, \ldots\right.$, $\left.\gamma_{m}\right)$ be a sequence of non-negative integers with sum ab. Suppose $\operatorname{rot}_{m}^{d}(\gamma)=\gamma$ for some $d \mid m$. Then

$$
\left(\operatorname{SSYT}\left(a^{b}, \gamma\right),\left\langle\partial^{d}\right\rangle, q^{\frac{1}{2}\left(a^{2} b-\left(\gamma_{1}^{2}+\ldots+\gamma_{m}^{2}\right)\right)} K_{a^{b}, \gamma}(q)\right)
$$

is a CSP-triple (Note that $\left\langle\partial^{d}\right\rangle$ is a cyclic group of size $m / d$ ). 
Note that if $\operatorname{rot}_{m}^{d}(\gamma)=\gamma$ this means $\gamma$ is the concatenation of $m / d$ copies of its first $d$ parts. Set $\mu:=\left(\gamma_{1}, \ldots, \gamma_{j d}\right)$. For example when $\gamma=12121212, d=2$ and $j=2$ we get $\mu=1212$. We will do a light abuse of notation here and say $\mu=\gamma^{j d / m}$.

Let $a^{b}$ be a fixed rectangular shape and $\gamma=\left(\gamma_{1}, \ldots, \gamma_{m}\right)$ be a composition of size $a b$ Combining the above result with Theorem 33 gives us the following identity for any $d \geq 1$ and $j \mid \frac{m}{d}$.

$$
\left|\left\{T \in \operatorname{SSYT}\left(a^{b}, \gamma\right): \partial^{j d}(T)=T\right\}\right|= \begin{cases}K_{a^{b}, \gamma^{j d / m}}^{(m /(j d))} & \text { if } \operatorname{rot}_{m}^{d}(\gamma)=\gamma \\ 0 & \text { otherwise }\end{cases}
$$

Let $\ell=\frac{m}{j d}$ and $\xi$ be a primitive $\ell^{\text {th }}$ root of unity. From (19) and Theorem 34 (with the assumption that $\left.\operatorname{rot}_{m}^{d}(\gamma)=\gamma\right)$ we have

$$
K_{a^{b}, \gamma^{1 / \ell}}^{(\ell)}=\xi^{\frac{1}{2}\left(a^{2} b-\left(\gamma_{1}^{2}+\ldots+\gamma_{m}^{2}\right)\right)} K_{a^{b}, \gamma}(\xi)
$$

due to the CSP. By Theorem 33, $K_{a^{b}, \gamma^{1 / \ell}}^{(\ell)}=\varepsilon_{\ell}\left(a^{b}\right)(-1)^{\left|\gamma^{1 / \ell}\right|(\ell-1)} K_{a^{b}, \gamma}(\xi)$, so (unless both sides vanish)

$$
(-1)^{\frac{a b}{\ell}(\ell-1)} \varepsilon_{\ell}\left(a^{b}\right)=\xi^{\frac{1}{2}\left(a^{2} b-\left(\gamma_{1}^{2}+\ldots+\gamma_{m}^{2}\right)\right)} .
$$

Note that we must have $\ell \mid a b$ in order for $K_{a^{b}, \gamma^{1 / \ell}}^{(\ell)}$ to be non-zero, so $(-1)^{\frac{a b}{\ell}(\ell-1)}$ is either -1 or 1 . Moreover, $\gamma_{1}^{2}+\ldots+\gamma_{m}^{2}=\ell\left(\gamma_{1}^{2}+\ldots+\gamma_{j d}^{2}\right)$, so there is a factor of $\ell / 2$ in the exponent of $\xi$.

Intuitively, we can then think that $\xi^{\gamma_{1}^{2}+\ldots+\gamma_{m}^{2}}=(-1)^{\gamma_{1}^{2}+\ldots+\gamma_{j d}^{2}}=(-1)^{\gamma_{1}+\ldots+\gamma_{j d}}=$ $(-1)^{\frac{a b}{\ell}}$. Hence, the appearance of $\gamma$ in $(21)$ is only used to define a "nice" exponent, where the dependence on $\ell$ is encapsulated in $\xi$.

Corollary 35. Given positive integers $a, b$ and $M$, where $M \mid a b$, there is an integer $E=E(a, b, M)>0$, such that for all $\ell \mid M$ with $\varepsilon_{\ell}\left(a^{b}\right) \neq 0$, we have

$$
(-1)^{\frac{a b}{\ell}(\ell-1)} \varepsilon_{\ell}\left(a^{b}\right)=\xi^{E},
$$

where $\xi$ is a primitive $\ell^{\text {th }}$ root of unity.

Proof. Using (21), we can choose $m$ and $d$ such that $M=\frac{m}{d}$ and a $\gamma$, satisfying $\operatorname{rot}_{m}^{d}(\gamma)=\gamma, \sum \gamma_{i}=a b$

We shall now use Corollary 35 to study shapes which are disjoint unions of rectangles. For positive integer vectors $\mathbf{a}=\left(a_{1}, \ldots, a_{r}\right), \mathbf{b}=\left(b_{1}, \ldots, b_{r}\right)$, let

$$
\mathbf{a}^{\mathbf{b}}:=\left(a_{1}^{b_{1}}\right) \oplus \ldots \oplus\left(a_{r}^{b_{r}}\right)
$$

denote a skew shape consisting of a disjoint union of $r$ rectangles, where rectangle $k, 1 \leq k \leq r$, has shape $a_{k}^{b_{k}}$. 
Lemma 36. Let $\mathbf{a}^{\mathbf{b}}$ be a disjoint union of rectangles, and $\gamma$ be a composition of length $m$ and size $a_{1} b_{1}+\ldots+a_{r} b_{r}$, with $\operatorname{rot}_{m}^{d}(\gamma)=\gamma$ for some $d \mid m$. There exists an $E \in \mathbb{N}$ (depending on $\mathbf{a}^{\mathbf{b}}, m$ and $d$ ), such that for all $j \mid \frac{m}{d}$ where $K_{\mathbf{a}^{\mathbf{b}}, \gamma}(\xi) \neq 0$,

$$
\xi^{E}=\operatorname{sgn} K_{\mathbf{a}^{\mathbf{b}}, \gamma}(\xi),
$$

where $\xi$ is a primitive $j^{\text {th }}$ root of unity.

Proof. First of all, Theorem 33 implies that unless $j$ divides $a_{k} b_{k}$ for all $k \in$ $\{1, \ldots, r\}$, we have $K_{\mathbf{a}^{\mathbf{b}}, \gamma}(\xi)=0$ where $\xi$ is a primitive $j^{\text {th }}$ root of unity. Hence, it suffices to consider the case when

$$
\frac{m}{d} \quad \text { divides } \operatorname{gcd}\left(a_{1} b_{1}, a_{2} b_{2}, \ldots, a_{r} b_{r}\right) .
$$

By Theorem 33 and some rewriting, we have

$$
\begin{aligned}
\operatorname{sgn} K_{\mathbf{a}^{\mathbf{b}}, \gamma}(\xi) & =(-1)^{\frac{1}{j}|\gamma|(j-1)} \varepsilon_{j}\left(\mathbf{a}^{\mathbf{b}}\right) \\
& =(-1)^{\frac{1}{j}\left|a_{1} b_{1}+\ldots+a_{r} b_{r}\right|(j-1)} \prod_{k=1}^{r} \varepsilon_{j}\left(a_{k}^{b_{k}}\right) \\
& =\prod_{k=1}^{r}(-1)^{\frac{a_{k} b_{k}}{j}(j-1)} \varepsilon_{j}\left(a_{k}^{b_{k}}\right) .
\end{aligned}
$$

We can now use Corollary 35- since we assume (24) - on each of the factors in the right hand side, and deduce that $\operatorname{sgn} K_{\mathbf{a}^{\mathbf{b}}, \gamma}(\xi)=\xi^{E_{1}+\ldots+E_{r}}=\xi^{E}$ for some fixed $E$ which does not depend on $j$, but only on $\mathbf{a}^{\mathbf{b}}, m$ and $d$.

We can now prove the main result of this subsection.

Theorem 37. (Cyclic sieving on disjoint rectangles) Let $\gamma=\left(\gamma_{1}, \ldots, \gamma_{m}\right)$ be a composition of size $a_{1} b_{1}+\ldots+a_{r} b_{r}$, satisfying $\operatorname{rot}_{m}^{d}(\gamma)=\gamma$. Then there exists some $E=E\left(\mathbf{a}^{\mathbf{b}}, \gamma\right) \in \mathbb{N}$ such that

$$
\left(\operatorname{SSYT}\left(\mathbf{a}^{\mathbf{b}}, \gamma\right),\left\langle\partial^{d}\right\rangle, q^{E} K_{\mathbf{a}^{\mathbf{b}}, \gamma}(q)\right)
$$

is a CSP-triple.

Before proceeding with the proof, let us briefly discuss some details. Promotion rotates the content, $\gamma$, so in order for $\partial^{d}$ to fix an element, it is trivially necessary that $\gamma$ is fixed under $\operatorname{rot}_{m}^{d}$. However, since promotion acts independently on each rectangle, this can only happen if each $\nu^{(k)}$ has rotational symmetry as well.

Proof. Let $\xi$ be a primitive $j^{\text {th }}$ root of unity, where $j \mid \frac{m}{d}$. As $\gamma$ has rotational symmetry, it is the concatenation of $m / d$ copies of $\mu:=\left(\gamma_{1}, \ldots, \gamma_{j d}\right)=\gamma^{j d / m}$. 
By Lemma 36, there is an $E>0$, depending only on $\mathbf{a}^{\mathbf{b}}$ and $\gamma$, such that $q^{E} K_{\mathbf{a}^{\mathbf{b}}, \gamma}(q)$ is a non-negative integer. Hence $\xi^{E} K_{\mathbf{a}^{\mathbf{b}}, \gamma}(\xi)$ is equal to $K_{\mathbf{a}^{\mathbf{b}}, \mu}^{(m /(j d))}$, and it remains to show that

$$
K_{\mathbf{a}^{\mathbf{b}}, \mu}^{(m /(j d))}=\left|\left\{T \in \operatorname{SSYT}\left(\mathbf{a}^{\mathbf{b}}, \gamma\right): \partial^{j d}(T)=T\right\}\right| .
$$

Since $\partial$ acts on each rectangle independently, the right hand side of (25) is given by

$$
\sum_{\nu^{(1)}+\ldots+\nu^{(r)}=\gamma} \prod_{k=1}^{r}\left|\left\{T \in \operatorname{SSYT}\left(a_{k}^{b_{k}}, \nu^{(k)}\right): \partial^{j d}(T)=T\right\}\right|,
$$

as we need to distribute the entries in $\gamma$ among the different rectangles. However, the product is 0 unless each composition $\nu^{(i)}$ has the rotational symmetry $\operatorname{rot}_{m}^{j d}\left(\nu^{(i)}\right)=$ $\nu^{(i)}$, so we have

$$
\sum_{\rho^{(1)}+\ldots+\rho^{(r)}=\mu} \prod_{k=1}^{r}\left|\left\{T \in \operatorname{SSYT}\left(a_{k}^{b_{k}},\left(\rho^{(k)}\right)^{m /(j d)}\right): \partial^{j d}(T)=T\right\}\right| .
$$

Now, the left hand side of (25) is given by

$$
\sum_{\rho^{(1)}+\ldots+\rho^{(r)}=\mu} \prod_{k=1}^{r} K_{a_{k}^{b_{k}, \rho^{(k)}}}^{(m /(j d))}
$$

since any skew semistandard ribbon tableau of shape $\mathbf{a}^{\mathbf{b}}$ and content $\mu$ is formed by $r$ semistandard ribbon tableaux of rectangular shape, where the total content is $\mu$. We can now see that (26) and (27) agree since by Theorem 33, for every $k \in\{1, \ldots, r\}$,

$$
\left|\left\{T \in \operatorname{SSYT}\left(a_{k}^{b_{k}},\left(\rho^{(k)}\right)^{m /(j d)}\right): \partial^{j d}(T)=T\right\}\right|=K_{a_{k}^{b_{k}}, \rho^{(k)}}^{(m /(j d)} .
$$

Hence, we have proved the CSP.

In the above proof, we were able to adjust the sign of $K_{\mathbf{a}^{\mathbf{b}}, \gamma}(q)$, by multiplying with an appropriate power of $q$, so that the result is a CSP-polynomial. The following example illustrates that adjusting the sign of a potential CSP-polynomial is not always possible.

Example 38. Let

$$
\left\{\begin{array}{l}
f(q)=6+2 q+3 q^{2}+2 q^{3}+3 q^{4}+2 q^{5} \\
g(q)=4+3 q+4 q^{2}+4 q^{4}+3 q^{5}
\end{array}\right.
$$


If we let $\xi$ be a primitive $6^{\text {th }}$ root of unity, then

$$
\begin{aligned}
\left(f\left(\xi^{1}\right), f\left(\xi^{2}\right), f\left(\xi^{3}\right), f\left(\xi^{6}\right)\right) & =(3,3,6,18) \\
\left(g\left(\xi^{1}\right), g\left(\xi^{2}\right), g\left(\xi^{3}\right), g\left(\xi^{6}\right)\right) & =(3,-3,6,18) .
\end{aligned}
$$

One can show that there is some $X$ of cardinality 18 , such that $\langle X, \mathbb{Z} / 6 \mathbb{Z}, f(q)\rangle$ is a CSP-triple. However, there is no $E \in \mathbb{Z}$ such that $q^{E} g(q)$ is a non-negative integer at every $6^{\text {th }}$ root of unity.

\section{Bi-cyclic sieving on ribbon SYT}

A natural generalization of CSP is when the product of two cyclic groups act simultaneously on the set. The bicycling phenomenon that was first considered in [BRS08] can be defined as follows: Assume we have two cyclic groups $C_{1}, C_{2}$ with generators $c_{1}, c_{2}$ of orders $k_{1}, k_{2}$ respectively, acting on a finite set $X$. Let $f(q, t)$ be a bivarite polynomial and $\zeta_{1}, \zeta_{2}$ be primitive $k_{1}$ and $k_{2}$-roots of unity. The triple $\left(X, C_{1} \times C_{2}, f(q, t)\right)$ is said to exhibit the bicyclic sieving phenomenon, biCSP for short, if for any $i, j \in \mathbb{Z}$ we have

$$
f\left(\zeta_{1}^{i}, \zeta_{2}^{j}\right)=\left|\left\{x \in X: c_{1}^{i} c_{2}^{j} \cdot x=x\right\}\right|
$$

That is, we have cyclic sieving for both cyclic groups, not only separately but also jointly. We prove biCSP for two families of ribbon SYT in this subsection.

Let $\mathrm{SYT}_{R}\left(\alpha_{1}, \ldots, \alpha_{\ell}\right)$ denote the set of ribbon standard Young tableaux with $\alpha_{i}$ boxes in row $i$.

Remark 39. The cardinality of $\operatorname{SYT}_{R}(m-b, b)$ is $\left(\begin{array}{c}m \\ b\end{array}\right)-1$, as there are $\left(\begin{array}{c}m \\ b\end{array}\right)$ ways to choose the second row, and all choices but $1,2, \ldots, b$ give a valid standard filling.

Theorem 40. The action $\partial$ on $\mathrm{SYT}_{R}(m-b, b)$ has a unique orbit of size $m-1$. All other orbits have sizes dividing $m$. In particular,

$$
\left(\mathrm{SYT}_{R}(m-b, b),\left\langle\partial^{m}\right\rangle,\left(\begin{array}{c}
m \\
b
\end{array}\right)-m+[m-1]_{q}\right)
$$

and

$$
\left(\mathrm{SYT}_{R}(m-b, b),\left\langle\partial^{m-1}\right\rangle,\left[\begin{array}{c}
m \\
b
\end{array}\right]_{q}-[m]_{q}+n-1\right)
$$

are CSP-triples.

Proof. Let us consider the action of promotion on a ribbon. Let $w=w_{1} \ldots w_{m}$ be the reading word. Either $w_{1}=1$ or $w_{b+1}=1$. If $w_{1}=1$ or if $w_{b+1}=1$ and $w_{b}<w_{b+2}$, 
promotion acts separately on each row.

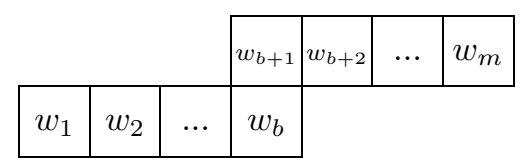

Only if $w_{b+1}=1$ and $w_{b+2}<w_{b}$ an exchange happens between rows, and that is only possible when the first row is $1, b+2, b+3, \ldots, m$. Let us call this particular tableau $T^{*}$. In the figure below we will use the notation $a:=m-b$ for convenience. The orbit of $T^{*}$ is of size $m-1$ :

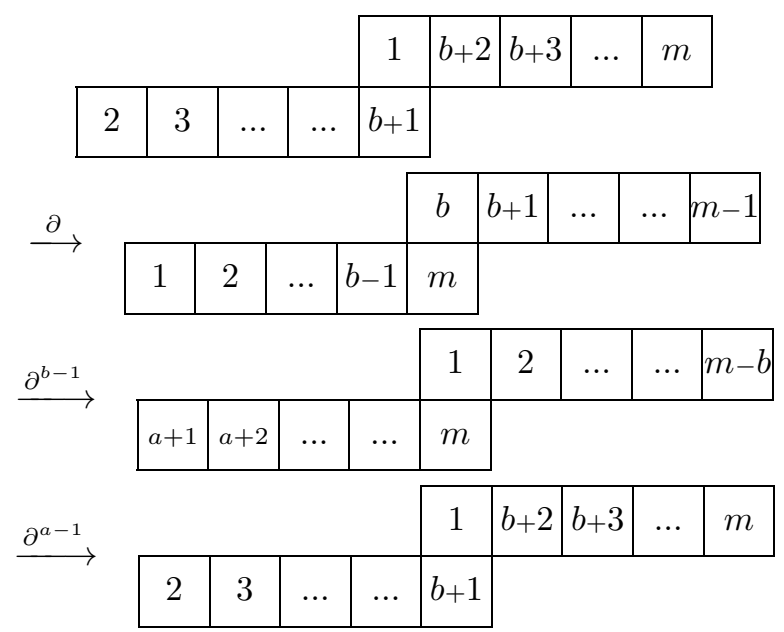

In any other orbit, there is no exchange between the rows, so promotion affects them independently. As each row is a rectangular tableaux and our alphabet has $m$ elements, the order of promotion divides $m$ for those orbits.

The first CSP follows as $\left\langle\partial^{m}\right\rangle$ has order $m-1$, and we have one orbit of size $m-1$, plus $\left(\begin{array}{c}m-1 \\ b\end{array}\right)-m$ elements that are fixed by everything.

For the second CSP, note that $\partial^{m-1}$ fixes the $(m-1)$-cycle described above. For the rest of the tableaux, the action of $\partial^{m-1}$ on the first row matches the rotation action on $b$ element subsets of $m$, except that we are missing the elements belonging to the $(m-1)$-cycle and the subset $\{m-b+1, m-b+2, \ldots, m\}$ which violates the tableaux rules.

Note that under the rotation action these elements actually form an $m$-cycle whose fixed points can be calculated by evaluating $[\mathrm{m}]_{q}$ at the appropriate root of unity. Subtracting this from the polynomial $\left[\begin{array}{c}m \\ b\end{array}\right]_{q}$ given by the CSP of rotation and adding back the $m-1$ fixed points gives us the desired result.

These two results can be combined to give a CSP for the action of $\partial$, but we will instead give a bicyclic version as the polynomial is nicer. 
Corollary 41. Promotion acting on $\mathrm{SYT}_{R}(m-b, b)$ has order $m(m-1)$. Let $\psi_{m, b}(q, t):=[m-1]_{q}+\left(\begin{array}{c}m \\ b\end{array}\right)_{t}-[m]_{t}$. Take a primitive $(m-1)^{\text {th }}$ root of unity $\xi$, and a primitive $m^{\text {th }}$ root of unity $\zeta$. Then for all $r, s \in \mathbb{Z}$,

$$
\psi_{m, b}\left(\xi^{r}, \zeta^{s}\right)=\left|\left\{T \in \mathrm{SYT}_{R}(m-b, b): \partial^{r m+s(m-1)}(T)=T\right\}\right| .
$$

In other words,

$$
\left(\mathrm{SYT}_{R}(m-b, b),\left\langle\partial^{m}\right\rangle \times\left\langle\partial^{m-1}\right\rangle,\left(\begin{array}{c}
m \\
b
\end{array}\right)_{t}-[m]_{t}+[m-1]_{q}\right)
$$

exhibits the bi-cyclic sieving phenomenon.

If $b=1$ or $m-1$, the total number of tableaux is $m-1$, so the $(m-1)$-orbit is the only one. If $b=m-b=2$, we have 5 tableaux in total, which are divided into a 3 -orbit and a 2 -orbit, so the promotion has order 6 . Next, we show that apart from these trivial cases, promotion on two rows has order $m(m-1)$.

Proposition 42. If $b, m-b>1$ and we do not have $b=m-b=2, \partial$ on $\mathrm{SYT}_{R}(m-b, b)$ has order $m(m-1)$.

Proof. We will show that in these cases, there is always an orbit of size $m$. Consider the tableaux with bottom row $1,2, \ldots, b-1, b+1$. This tableau does not come up in the $(m-1)$-cycle, so promotion is applied independently to the two rows. As promotion has order $m$ on the bottom row, it has order $m$ on the tableau.

Note that the same arguments apply to the two column case by symmetry. Next, we consider the three row ribbon case where the first and last rows consist of one box only.

Lemma 43. We have that $\left|\mathrm{SYT}_{R}(1, m-2,1)\right|=(m-1)(m-2)-1$.

Proof. There are $m-1$ ways to pick the entry that goes on the first row, as it can not be $m$, and $m-2$ ways to pick the entry in the last row so that it won't be the smallest entry of the rest. Of these $(m-1)(m-2)$ choices, only one does not give a tableau - choosing $m-1$ for top row and $m$ for the bottom row.

Theorem 44. For $m>3$, promotion acting on $\mathrm{SYT}_{R}(1, m-2,1)$ has order $(m-1)(m-2)$, with one $(m-2)$-cycle and $m-3(m-1)$-cycles. As a consequence, if we let $\psi_{m, b}(q, t):=[m-2]_{t}+(m-3)[m-1]_{q}$, $\xi$ be a primitive $(m-1)^{\text {th }}$ root of unity and $\zeta$ be a primitive $(m-2)^{\text {th }}$ root of unity, for all $r, s \in \mathbb{Z}$ we have

$$
\psi_{m, b}\left(\xi^{r}, \zeta^{s}\right)=\left|\left\{T \in \operatorname{SYT}_{R}(1, m-2,1): \partial^{r(m-2)+s(m-1)}(T)=T\right\}\right| .
$$

In other words,

$$
\left(\mathrm{SYT}_{R}(1, m-2,1),\left\langle\partial^{m-1}\right\rangle \times\left\langle\partial^{m-2}\right\rangle,[m-2]_{t}+(m-3)[m-1]_{q}\right)
$$

exhibits the bi-cyclic sieving phenomenon. 
Proof. There are $m-2$ tableaux where the top row is 1 . If the bottom row is $m$, we get the following $m-2$ cycle:

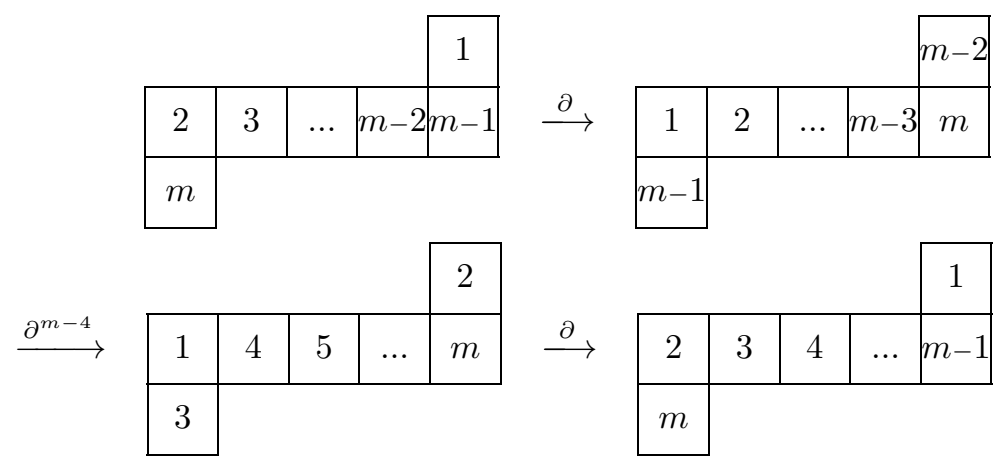

For the other $m-3$ cases where bottom row contains $k<m$ we get the following $m-1$ cycles (we denote $m-k$ by $a$ for visual clarity):

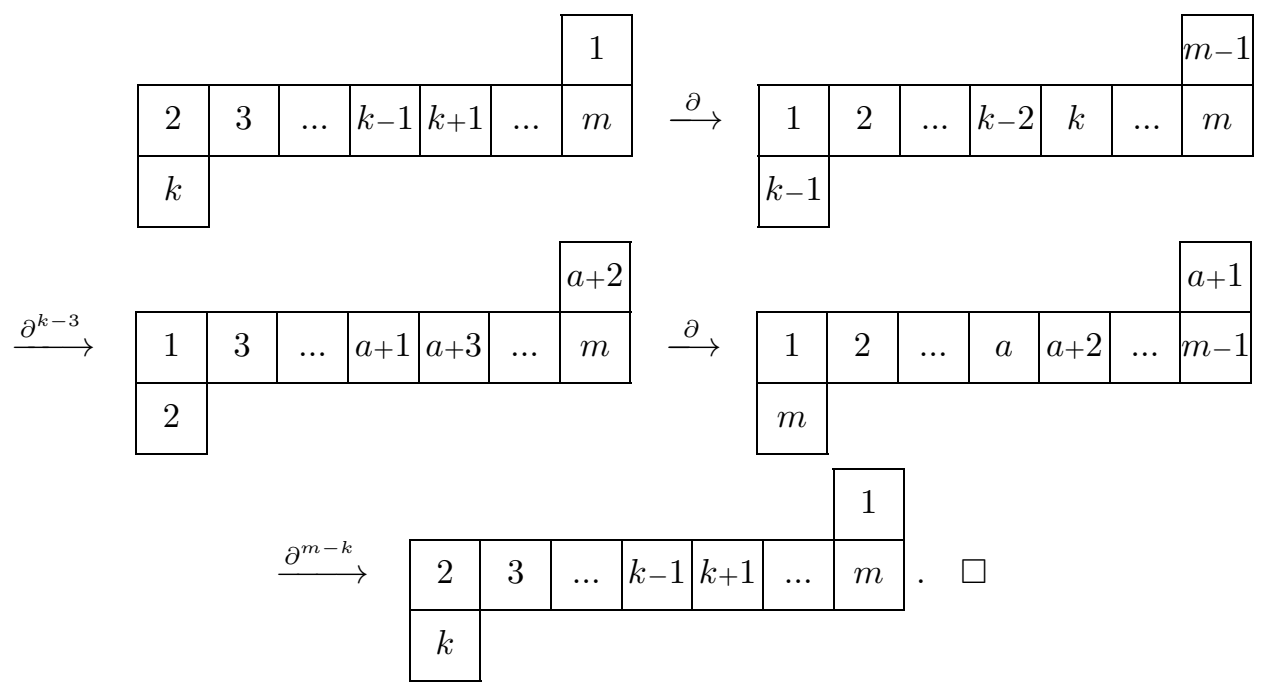

\section{Discussion}

We have covered promotion on a few new classes of shapes, and in particular, paid some well-deserved attention to skew shapes. In many cases, the KostkaFoulkes polynomials play a central role, except in the last section where we needed something different.

It is natural to examine other families of ribbon shapes. However, the order of promotion on the set $\mathrm{SYT}_{R}(k, k, k)$ for $k=1, \ldots, 4$ is $1,60,814773960$, and 
82008289440, respectively, which is discouraging. Similarly, the order of promotion on the set $\operatorname{SYT}_{R}(1,1, k, 1)$ for $k \geq 1$ seems to be (we have verified this for $k \leq 15$ ) given by the generating function

$$
x \frac{1+16 x-19 x^{2}+10 x^{3}-2 x^{4}}{(1-x)^{4}}=x+20 x^{2}+55 x^{3}+114 x^{4}+203 x^{5}+\ldots
$$

The main interesting open case is the staircase shape $s c_{m}:=(m, m-1, \ldots, 2,1)$, where the promotion action has order $m(m-1)$ (see [Hai92]). The possibility of obtaining a CSP triple was first considered in [PW11]; the authors gave an injection from $\operatorname{SYT}\left(s c_{m}\right)$ to $\operatorname{SYT}\left(m^{m+1}\right)$ that commutes with promotion. However, there are no good candidate polynomials conjectured to provide instances of CSP for general $m$.

Problem 45. Find some $p_{m}(q) \in \mathbb{N}[q]$ such that

$$
\left(\mathrm{SYT}\left(s c_{m}\right),\langle\partial\rangle, p_{m}(q)\right)
$$

is a CSP-triple.

Promotion on the shifted staircase admits a CSP; this is an example of a minuscule poset which behaves nicely with respect to promotion and cyclic sieving, see [RS12]. For more background and discussion, see [Hop20b].

In [Hop20a, Conjecture 5.2], a related problem regarding cyclic sieving on triangular plane partitions is stated, with an explicit polynomial.

Finally, a different approach would be to keep the Kostka-Foulkes polynomials, and replace promotion with a different group action. In [APRU20], it is shown that the set of standard Young tableaux of shape $m \lambda:=\left(m \lambda_{1}, m \lambda_{2}, \ldots, m \lambda_{\ell}\right)$ admits a cyclic sieving phenomenon with $K_{m \lambda, 1|m \lambda|}(q)$ as the CSP-polynomial and some cyclic group of order $m$.

As a more concrete open problem, the statement of Theorem 37 with $h\left(\mathbf{a}^{\mathbf{b}}, \gamma\right)$ is a bit unintuitive, as there is a choice of $\delta^{(j)}$ 's involved.

Problem 46. Is there some way to define $h\left(\mathbf{a}^{\mathbf{b}}, \gamma\right)$ without this seemingly arbitrary choice?

\section{References}

[AKlm05] Abuzzahab, O., Korson, M., Li Man Chun, M. and Meyer, S., Cyclic and dihedral sieving for plane partitions, U. Minnesota REU Report, 2005. http://www-users.math.umn.edu/ reiner/REU/ PlanePartitionReport.pdf. 
[APru20] Alexandersson, P., Pfannerer, S., Rubey, M. and Uhlin, J., Skew characters and cyclic sieving, Preprint, 2020. arXiv:2004.01140. To appear in SIGMA.

[BMS14] Bennett, M., Madill, B. and Stokke, A., Jeu-de-taquin promotion and a cyclic sieving phenomenon for semistandard hook tableaux, Discrete Math. 319 (2014), 62-67. doi:10.1016/j .disc. 2013.11.024

[BRS08] Barcelo, H., Reiner, V. and Stanton, D., Bimahonian distributions, J. Lond. Math. Soc. 77 (2008), 627-646. doi:10.1112/jlms/jdn004

[But94] Butler, L. M., Subgroup Lattices and Symmetric Functions, Am. Math. Soc., Providence, 1994. https://bookstore.ams.org/memo-112-539

[DLT94] Désarménien, J., Leclerc, B. and Thibon, J.-Y., Hall-Littlewood functions and Kostka-Foulkes polynomials in representation theory, Sémin. Lothar. Comb. [electronic only] 32 (1994), 38. http: //eudml .org/doc/ 119019

[FK13] Fontaine, B. and Kamnitzer, J., Cyclic sieving, rotation, and geometric representation theory, Sel. Math. New Ser. 20 (2013), 609-625. doi:10 . 1007/s00029-013-0144-4

[Hai92] Haiman, M. D., Dual equivalence with applications, including a conjecture of Proctor, Discrete Math. 99 (1992), 79-113. doi:10 . 1016/0012-365x(92) 90368-p

[Hop20a] Hopkins, S., Cyclic sieving for plane partitions and symmetry, SIGMA (2020). doi:10.3842/sigma. 2020.130

[Hop20b] Hopkins, S., Order polynomial product formulas and poset dynamics. Preprint, 2020. arXiv:2006.01568

[KB96] Kirillov, A. N. and Berenstein, A. D., Groups generated by involutions, Gelfand-Tsetlin patterns, and combinatorics of Young tableaux, St. Petersburg Math. J. 7 (1996), 77-127. Algebra i Analiz 7 (1995), 92152. http://mi.mathnet.ru/eng/aa491

[Mac96] Macmahon, P. A., Memoir on the theory of the partition of numbers. Part I, Philos. Trans. R. Soc., Math. Phys. Eng. Sci. 187 (1896), 619-673. doi:10.1098/rsta.1896.0016

[OP19] Он, Y.-T. and PARK, E., Crystals, semistandard tableaux and cyclic sieving phenomenon, Electron. J. Comb. 26 (2019). doi:10. 37236/8802

[PSV16] Pressey, T., Stokke, A. and Visentin, T., Increasing tableaux, Narayana numbers and an instance of the cyclic sieving phenomenon, Ann. Comb. 20 (2016), 609-621. doi:10 . 1007/s00026-016-0320-2

[PW11] Pon, S. and WAng, Q., Promotion and evacuation on standard Young tableaux of rectangle and staircase shape, Electron. J. Comb. 18 (2011). doi:10.37236/505

[Rho10a] Rhondes, B., Cyclic sieving, promotion, and representation theory, J. Comb. Theory, Ser. A 117 (2010), 38-76. doi:10.1016/j . jcta. 2009.03.017

[Rho10b] RhoAdes, B., Hall-Littlewood polynomials and fixed point enumeration, Discrete Math. 310 (2010), 869-876. doi:10.1016/j.disc.2009.10.003

[RS12] Rush, D. B. and SHI, X., On orbits of order ideals of minuscule posets, J. Algebraic Comb. 37 (2012), 545-569. doi:10.1007/s10801-012-0380-2

[RSW04] Reiner, V., Stanton, D. and White, D. E., The cyclic siev- 
ing phenomenon, J. Comb. Theory, Ser. A 108 (2004), 17-50. doi:10.1016/j.jcta. 2004.04.009

[Sch63] SchütZEnBerger, M. P., Quelques remarques sur une construction de Schensted, Math. Scand. 12 (1963), 117. doi:10.7146/math. scand . a-10676

[Sch72] Schützenberger, M. P., Promotion des morphismes d'ensembles ordonnes, Discrete Math. 2 (1972), 73-94. doi:10.1016/0012-365x (72) 90062-3

[Sta01] Stanley, R. P., Enumerative Combinatorics: Volume 2, 1st ed., Cambridge University Press, Cambridge, 2001. doi:10.1017/CB09780511609589

[SW12] Striker, J. and Williams, N., Promotion and rowmotion, Eur. J. Comb. 33 (2012), 1919-1942. doi:10.1016/j . ejc.2012.05.003

[SW20] Shen, L. and Weng, D., Cyclic sieving and cluster duality of Grassmannian, SIGMA (2020). https ://doi.org/10.3842/sigma.2020.067

Per Alexandersson

Department of Mathematics

Stockholm University

Stockholm SE-10691

Sweden

per.w.alexandersson@gmail.com

Received August 20, 2020

in revised form February 23, 2021
Ezgi Kantarci Oğuz

Dept. of Mathematics

KTH-Royal Institute of Technology

Stockholm SE-100 44

Sweden

ezgikantarcioguz@gmail.com

Svante Linusson

Dept. of Mathematics

KTH-Royal Institute of Technology

Stockholm SE-100 44

Sweden

linusson@kth.se 